\title{
El estudio neurológico de la conciencia: Una valoración crítica
}

\author{
José M. Zumalabe-Makirriain
}

Facultad de Psicología de la Universidad del País Vasco/Euskal Herriko Unibertsitatea (España).

\begin{abstract}
Resumen: Durante el predominio, en ciencia cognitiva, del paradigma clásico simbólico-computacional no se prestó apenas atención al estudio neurobiológico de la conciencia. Con la aparición de los modelos conexionistas en los que se parte de una concepción más naturalizada y menos "abiológica” de la psicología, se dio un giro radical en la cuestión que nos ocupa. Desde esta corriente la explicación de los fenómenos mentales debe incluir necesariamente referencias al soporte biológico ya que se toma como modelo computacional el funcionamiento del sistema nervioso. Tras describir los trabajos de los neurocientíficos sobre los correlatos neuronales de la conciencia, analizamos desde una perspectiva crítica, las limitaciones y puntos débiles de los mismos referidas fundamentalmente a los problemas conceptuales del conexionismo, a las objeciones al localizacionismo, a las limitaciones de las técnicas de imagen cerebral, a la falta del establecimiento de relaciones causales cerebro-mente y a la pretensión exclusivista y reduccionista de la mayor parte de las investigaciones.

Palabras clave: conciencia; neurociencias; conexionismo; técnicas de neuroimagen; correlatos neuronales.
\end{abstract}

Title: The neurological study of the conscience: a critical assessment.

Abstract: During the predominance, in the cognitive science, of the classic symbolic-computational paradigm hardly was paid attention to the neurobiological study of the consciousness. With the appearance of the connectionist models which start of a more naturalized and less abiologic conception of the psychology, was taken a radical turn in this subject. Since this current the explanation of the mental phenomenons needs to include references to the biological support because is taken like a computational model the running of the nervous system. After describing the works of the neuroscientist about the neuronal correlates on the consciousness, we analyze since a critical perspective, the limitations and the weaknesses of them refered basically to the conceptual troubles of the connectionism, to the objections to the localizacionism, to the limitations of the cerebral image techniques, to the lack of stablishment of the brain-mind causal relations and to the exclusivist and reductionist pretension of the most part of the investigations.

Key words: consciousness; neuroscience; connectionism; neuroimage thecniques; neuronal correlates.

la ciencia cognitiva pueden identificarse respectivamente con el paradigma clásico simbólico-computacional y con la alternativa conexionista.

A pesar de que la opción neurológica era más realista y aportaba una cierta solución no dualista al problema de la relación entre el cuerpo y la mente computacional, fue la opción simbólico-computacional la predominante en el cognitivismo desde finales de los años sesenta hasta mediados de los ochenta del pasado siglo, cuando el conexionismo retomó la opción neural. Posiblemente sea más realista una concepción más naturalizada de la psicología que la orientación abiológica de la psicología cognitiva simbólicocomputacional para la que, el hecho de que la mente se materialice en un cerebro era algo accidental ya que lo importante eran las relaciones en el nivel funcional.

La investigación neurológica de la conciencia durante décadas se vio obstaculizada por la creencia generalizada de que la conciencia es sólo un tipo especial de programa computacional, es decir un "software" especial en el "hardware" del cerebro; sería sólo una cuestión de procesamiento de información. Se trataba de una perspectiva, en la que el estudio del cerebro quedaba al margen. Hoy los planteamientos que defienden la independencia del cerebro y la mente están siendo cada vez más desautorizados desde el funcionalismo de la biología evolucionista, desde la neuropsicología cognitiva y desde las neurociencias cognitivas.

Cosmides y Tooby $(1995,2013)$ defienden que tiene que darse una relación causal entre la estructura de un mecanismo de procesamiento de información y su función ya que la selección natural opta por un diseño determinado sobre la base de cómo éste soluciona los problemas adaptativos. El cerebro es un sistema que se organiza siguiendo la lógica evolucionista de la adaptación a la funciones para la que fue diseñado, por lo que la estructura neural y la estructura cog-
* Dirección para correspondencia [Correspondence address]:
Facultad de Psicología de la Universidad del País Vasco/Euskal Herriko Unibertsitatea. Departamento de Personalidad, Evaluación y Tratamiento Psicológico. Avenida de Tolosa, 70. 20018 San Sebastián (España). E-mail: josemaria.zumalabe@ehu.es 
nitiva están íntimamente relacionadas. En consecuencia no tiene ningún sentido defender la independencia cerebromente, aunque éste pueda ser un planteamiento válido en el ámbito de los mecanismos artificiales de procesamiento de la información, se torna tan insuficiente como la propia metáfora del ordenador a la hora de explicar la actividad de un mecanismo biológico de procesamiento de información como el sistema cerebro/mente. “...hoy por hoy, el problema más importante en las ciencias biológicas es el problema de la conciencia ...comprender la naturaleza de la conciencia requiere, decisivamente, comprender cómo es que los procesos cerebrales causan y llevan a cabo la conciencia" (Searle, 2007, pp. 30-31).

Con el conexionismo se dio un giro radical en la cuestión que nos ocupa. Desde esta nueva corriente computacional, se considera que la explicación de los fenómenos mentales necesariamente debe incluir una referencia al soporte biológico; los fenómenos cognitivos se explican en función de la actividad de redes que se comportan como sistemas dinámicos no lineales enmarcados en una organización a gran escala del cerebro y el sistema nervioso (Rogers y McClelland, 2014). Ignorar las aportaciones de las neurociencias implicaría caer, otra vez, en el error que cometieron los primeros psicólogos cognitivos que supuso aceptar y defender la independencia entre el "software" (mente) y el "hardware" (cerebro), con las limitaciones que esto conllevaba.

Desde el conexionismo de segunda generación también conocido como el enfoque del Procesamiento Paralelo Distribuido (PPD) se asume que:

...el cerebro está constituido por un conjunto de elementos de cómputo (las neuronas) que efectúan operaciones simples e interactúan localmente a través de un conjunto de relaciones de conexión (uniones sinápticas) que pueden ser modificadas por mecanismos de aprendizaje y autoorganización. El cerebro es considerado como un sistema de procesamiento paralelo masivo que representa el conocimiento por medio de la actividad conjunta y distribuida de una población de neuronas (Martínez, 2006, p. 142).

Las computaciones no se realizan sobre expresiones simbólicas de carácter proposicional sino que son el producto de la interacción de numerosas unidades interconectadas, a ninguna de las cuales se les puede atribuir una significación independiente (McClelland, 2010; Rumelhart, McClelland, y PDP, 1986). El cerebro es el órgano de la mente y su particular relevancia procede de lo que el cerebro hace, no de lo que el cerebro es y lo que hace es generar la mente, es decir fabricar pensamientos, sentimientos y acciones. Aunque desde ciertas posiciones psicológicas hay quien atribuye al medio ambiente la capacidad de "crear mente", posiblemente sería más adecuado considerar que éste sólo tiene la capacidad de modularla con su interacción o de dotarla de los contenidos sociales y culturales correspondientes.

Los trabajos de investigación llevados a cabo desde los modelos conexionistas han revelado que la formalización matemática del procesamiento de la información puede aportar valiosas contribuciones para llegar a poner en claro tanto las propiedades computacionales del funcionamiento cerebral, como las bases analíticas que permitan formular las características de la representación neuronal de los contenidos de la experiencia consciente (McClelland, 2013). Ahora bien, aunque desde la representación distribuida y subsimbólica de las perspectivas conexionistas se hayan resuelto algunas de las limitaciones y puntos débiles del paradigma computacional clásico, la misma perspectiva conexionista conlleva una serie de problemas no resueltos todavía y que será necesario solventar para afrontar con éxito un análisis formal riguroso de la actividad consciente ya que es evidente la existencia de ciertas lagunas conceptuales que deben ser superadas para formular una teoría reduccionista de la conciencia desde esta perspectiva.

El nivel subsimbólico del conexionismo supone una clase de lógica alternativa al formalismo empleado por el computacionalismo clásico de la IA. Para entender los procesos mentales necesitamos conocer la función o el diseño cerebral, ya que como señalaron Kosslyn y Koenig (1992), en la línea de lo que siempre han mantenido los enfoques emergentistas, se puede equiparar la descripción de los eventos mentales con la descripción de la función del cerebro, afirmando que "la mente es lo que hace el cerebro" (p. 4). Desde este planteamiento, tanto las neurociencias como la psicología cognitiva, se proponen como objetivo general desarrollar una teoría de la actividad psicológica humana basada en las relaciones entre los procesos mentales y la estructura cerebral.

Desde la neurociencia se ha tratado de entender cómo el cerebro es capaz de producir la mente ya que los procesos mentales se consideran como propiedades emergentes de los procesos cerebrales (Gazzaniga y Heatherton, 2008; Gazzaniga y Mangun, 2014; Llinas, 2008). Posiblemente, en los últimos años, la neurociencia haya sido la disciplina que más ha contribuido a esclarecer el problema clásico de la relación entre la física y la química del cerebro con la conciencia del mundo que nos rodea.

Tradicionalmente las neurociencias han estado alejadas del estudio de los fenómenos mentales; partiendo del principio materialista de que la mente era un producto del cerebro, resultaba coherente creer que un profundo conocimiento del funcionamiento del cerebro, proporcionaría un conocimiento exhaustivo de la mente. Pero hoy las cosas han cambiado significativamente y el avance de las neurociencias ha proporcionado nuevas herramientas para enfocar estas cuestiones de otra manera. El trabajo sobre registro de la actividad neuronal unitaria posibilitado por las nuevas tecnologías está permitiendo explicar la vida mental desde el funcionamiento del cerebro.

Las nuevas tecnologías que permiten ver el cerebro en funcionamiento e identificar las áreas cerebrales implicadas en la realización de una tarea psicológica controlada, han posibilitado el surgimiento de nuevas líneas de investigación sobre la relación cerebro-mente y están revolucionando nuestro conocimiento sobre el funcionamiento del cerebro, “...la Neurociencia Cognitiva ya está permitiendo plantear 
cuestiones nuevas sobre la mente y el cerebro y, probablemente, también nuevos caminos para responderlas" (RuizVargas, 1999, p.15).

Roger Sperry (1913-1994), reconocido con el premio Nobel de Fisiología y Medicina, en 1981, por sus trabajos acerca de las funciones de los hemisferios cerebrales, afirmó que:

Las ciencias del comportamiento y del cerebro no eliminan los valores humanistas, como el de la libertad, ni tampoco dejan a la mente y a la conciencia como simples subproductos o epifenómenos de mecanismos cerebrales. Las ideas son tan reales como las neuronas y pueden tener fuerza causal. Mente y conciencia ocupan una posición de alto control sobre los procesos del cerebro (2000, p.1).

En la nueva situación, los estudios sobre la mente han pasado a un primer plano, aunque la perspectiva sea diferente a la del pasado, de la misma manera que se ha recuperado el estudio de la conciencia para la psicología aunque se haya reconceptualizado el término. Estas palabras de Roger Sperry, nos pueden servir para ubicar la cuestión:

En la nueva síntesis, los estados mentales, como propiedades dinámicas emergentes de la actividad cerebral, se encuentran inseparablemente fundidos con y vinculados a la actividad cerebral de la que son una propiedad emergente. La conciencia, según esta visión, no puede existir aparte del cerebro en funcionamiento (Sperry, 1993, p.880).

Son los procesos cerebrales los que causan las experiencias conscientes.; un estado consciente es un estado en el que está el cerebro, una característica de nivel superior del mismo cerebro, no una sustancia separada del mismo. "Así como el agua puede estar en estado líquido o sólido,...la conciencia es un estado en el que el cerebro se encuentra sin que por ello la conciencia sea una sustancia separada" (Searle, 2007, p. 15). Hoy existe un amplio acuerdo en que la conciencia es un fenómeno biológico causado por procesos cerebrales, lo que es más complicado es discernir cómo acontece esa causación.

Hace veinte años los neurocientíficos se resistían a estudiar el problema de la conciencia, pero hoy son muchos los trabajos que lo abordan con rigor. Todos ellos tratan de discernir la forma en que los procesos cerebrales causan los estados conscientes y cómo se llevan a cabo esos estados en las estructuras cerebrales (Capurro y Quian Quiroga, 2009; Greenfield, 2011; Koch, 2009, 2013; Koch y Tsuchiya, 2007; Navajas, Rey y Quian Quiroga, 2014; Sporns, 2013; Tononi y Koch, 2008; Quian Quiroga, 2008). Ese objetivo implica un interés acerca de cuáles y cómo son los correlatos neurobiológicos de la conciencia y de entre ellos cuáles son realmente los responsables causales de la producción de la conciencia.

\section{Los correlatos neuronales de la conciencia}

Se supone que los contenidos de la experiencia consciente están correlacionados con la actividad exhibida por un sistema neuronal, cuando, ante un evento específico, el sujeto informa verbalmente del estímulo en cuestión, y además, en su cerebro se detecta por cualquiera de los procedimientos disponibles, que hay un grupo de neuronas que dispara sistemáticamente con un patrón específico de potenciales de acción ante ese evento en particular. Ese contenido definido por los patrones neurales de activación se considera como una representación del contenido aparente que tiene para el sujeto la experiencia consciente de percibir el estímulo. Las características de activación espacial y el patrón temporal de disparo del grupo de neuronas involucradas en la percepción consciente determinarán la naturaleza de esta representación neuronal.

A diferencia de los modelos clásicos simbólicocomputacionales, los elementos básicos de significado o contenido semántico (primitivos representacionales) son diferentes de las unidades computacionales, y se presentan en forma distribuida a un nivel superior al de cómputo (Smolensky, 1988, 2012). Los primitivos computacionales, como las neuronas, por ejemplo, ejecutan operaciones simples que en sí mismas carecen de contenido semántico; a través del patrón de actividad conjunta de estos elementos, y de su paso por una amplia matriz de conexiones es como se llegan a constituir las unidades de significado para el sistema cognitivo (Chalmers, 2012). En este contexto, el cómputo opera a un nivel más fundamental que el de las representaciones, es por ello que Smolensky denomina subsimbólica a esta clase de computación.

Koch, (1996, 2009) sostiene que existe una representación simbólica de la realidad cuando se presenta un patrón de disparo de una neurona, o de un vector de actividad de una agrupación de neuronas, que tiene una fuerte correlación con una característica particular del mundo (orientación visual, movimiento, color, textura,...). El significado del simbolo neuronal va a depender del estatus del grupo de neuronas en la jerarquía de múltiples niveles que conforman el sistema, es decir, de la naturaleza de las neuronas en el grupo de células, de su campo receptivo y de su campo de estimulación, o lo que es lo mismo, de las neuronas en las que influye $\mathrm{y}$ de las que recibe influencia.

Un sistema de representación neuronal es un lenguaje o un código, mediante el que el cerebro representa la información acerca de las propiedades y los contenidos específicos del estímulo presentado que varían en función del tiempo por lo que la tarea de analizar este código es bastante compleja (Abbott \& Sejnowski, 1999; Gabbiani \& Koch, 1999; Libet, 2004). Estos sistemas representacionales, formalmente pueden ser conceptualizados por las descripciones cuantitativas que ofrecen las ecuaciones de conducción nerviosa que suponen el sustento biofísico de los modelos computacionales de la neurociencia experimental (Koch \& Segev, 1998; O’Reilly \& Munakata, 2000).

Superadas las reticencias del pasado, desde las neurociencias se ha abordado profusamente el estudio de los correlatos neurobiológicos de la conciencia (NCC por sus siglas en inglés). Crick y Koch (1990, 2007) consideran que la mejor manera de abordar el estudio de la conciencia es encontrar sus correlatos neuronales y las funciones cerebrales 
que dan lugar a las experiencias conscientes. Se trata de identificar los correlatos neuronales que son causalmente responsables de la producción de la conciencia y de descubrir los principios según los cuales los fenómenos biológicos (los disparos neuronales, por ejemplo), pueden ocasionar los estados subjetivos de sensación y alerta que caracterizan a la conciencia (Koch y Crick, 2001). También se trata de discernir si la conciencia es un fenómeno global de todo el cerebro o si se localiza en ciertas regiones específicas y si se tratara del segundo caso ¿en cuáles?

En este sentido, Koch defiende que para cada experiencia consciente, un grupo único de neuronas de regiones cerebrales concretas se activan de manera específica; sin embargo, Greenfield considera que la conciencia está generada por un incremento cuantitativo en la actividad holística del cerebro (Koch y Greenfield, 2007). Ambos neurocientíficos comparten, cada uno por su lado, el interés por buscar los correlatos neuronales de la experiencia consciente que puedan servir para deducir los mecanismos causales creadores de la conciencia, el primero considerando que las diferentes experiencias conscientes están mediadas por grupos específicos de neuronas (Crick \& Koch, 2003) y la segunda sosteniendo que para cada experiencia consciente, neuronas distribuidas por el cerebro se sincronizan en asambleas coordinadas, dispersándose luego (Greenfield, 2011; Greenfield y Collins, 2005).

Desde estas perspectivas se indaga acerca de cuál es el nivel apropiado para explicar la conciencia. Muchos autores se inclinan por el nivel de las neuronas y sinapsis; otros optan por niveles funcionales superiores como los mapas neuronales (Edelman, 1989, 1992, 2002, Izhikevich, Gally y Edelman, 2004) o nubes enteras de neuronas (Freeman, 1995); y otros orientan su atención por debajo del nivel de las neuronas y sinapsis hasta el nivel de los microtúbulos (Hameroff, 1998a, 1998b; Hameroff y Penrose, 2014; Penrose, 1994), mientras otros prefieren una perspectiva más global (Pribram, 1976, 1991, 1999). En definitiva, todos se proponen explicar cómo los procesos cerebrales, que son procesos objetivos biológicos, químicos y eléctricos, de tercera persona, producen estados subjetivos de sensación y pensamiento, es decir, de primera persona.

Desde una perspectiva muy general, por correlato neuronal de la conciencia se entiende el conjunto de eventos que se observan en el cerebro humano cuando se presenta un estado mental consciente que puede ser observado y medido con las diferentes técnicas de imagen cerebral. En los últimos años se han presentado gran número de teorías que van desde las que proponen que la relación concienciacerebro es una especie de paralelismo en el que dos reinos ontológicamente distintos (el físico y el mental) se sincronizan sin afectarse, hasta los planteamientos que presentan la experiencia consciente como un fenómeno material y físico.

Eccles (1980, 1989) neurofisiólogo australiano que fue reconocido con el Premio Nobel en 1963 por sus trabajos sobre el mecanismo iónico de excitación e inhibición de las sinapsis cerebrales, fue uno de los primeros autores en inten- tar explicar en términos de conciencia el problema de las relaciones cerebro-mente. Desde una postura dualista interaccionista Eccles considera que la conciencia subjetiva de los fenómenos mentales es inexplicable y se opone a cualquier intento científico de reducir la conciencia a actividad neuronal. En la misma línea, desde una posición fenomenológica Chalmers (1997, 1998, 2004, 2010) también afirma la irreductibilidad de la conciencia a actividades neuronales, planteando su conocida división entre el problema fácil y el problema duro de la conciencia.

Desde los diferentes naturalismos (Cotterill, 1998; Edelman, 1992, 2002) se defiende que una explicación completa de la mente y de la conciencia debe incluir sus bases y mecanismos biológicos o somáticos. Así, desde una posición naturalista biológica Damasio $(1996,1999,2000,2001)$ ha criticado la conceptualización por separado del cerebro y la conciencia por considerarla como un lastre del cartesianismo que todavía perdura, e insiste en el carácter biológico de la mente y la conciencia.

Decimos que un fenómeno es emergente cuando resulta (emerge) de la actividad de una serie de elementos constitutivos, pero que no está presente en los elementos constitutivos mismos. Por ejemplo, determinada textura resulta o emerge de cierta organización reticular de los átomos de un cuerpo, pero los átomos mismos no poseen esa textura.

Las teorías emergentistas actuales conceden mucha importancia a los fenómenos colectivos de interacción y organización de poblaciones de neuronas, así como a los fenómenos que resultan o emergen de ellos. Sus modelos explicativos se alejan del paradigma mecanicista clásico, tomando en consideración los fenómenos de auto-organización, complejidad, organización no lineal, comportamiento caótico, etcétera. Se propone, que la conciencia sea entendida como una propiedad que resulta o emerge de los patrones de actividad de las neuronas en redes neuronales sin que las neuronas mismas posean tal propiedad.

La mayor parte de los teóricos e investigadores en este ámbito han adoptado, tácita o explícitamente la denominada teoría de los bloques de la conciencia. Desde esta perspectiva se considera que cualquier campo consciente está constituido por pequeños componente que se combinan entre sí para formar el campo general de la conciencia (Zeki, 2007a). Si encontramos el NCC causal de cualquiera de los componentes habríamos encontrado un elemento causalmente necesario y suficiente para esa experiencia consciente, que extendido al resto de los campos nos permitiría descifrar el problema que nos ocupa.

Investigadores como Crick (1994), premio Nobel de Medicina y Fisiología en 1962 junto con Watson por su descubrimiento de la estructura del ADN, o Llinás (2001) intentan dar un amplio alcance explicativo a los eventos de oscilaciones sincronizadas; piensan que es probable que todos los aspectos de la conciencia empleen un mecanismo común básico en los humanos, o quizá unos cuantos mecanismos y que si logramos entender el mecanismo correspondiente para un aspecto, es posible que lo hayamos entendido para to- 
dos. Además sugieren que una de las funciones de la conciencia podría consistir en presentar el resultado de varias computaciones subyacentes, y que esto involucra a un mecanismo que une temporalmente a las neuronas mediante la sincronización de sus espinas dendríticas en oscilaciones de $40 \mathrm{~Hz}$. El evento celular que inicia y permite la sincronización de una neurona con el resto de la red se presenta en una región de la neurona especializada en la recepción de inputs provenientes de otras neuronas: la dendrita, más específicamente en los receptores de las espinas de las dendritas.

Crick y Koch $(1998,2007)$ plantean explícitamente que si pudiéramos descifrar la conciencia visual tendríamos la clave para descifrar toda la conciencia. La idea es que si encontramos el NCC de la visión, podríamos explicar la conciencia visual y sabríamos qué buscar para encontrar el NCC de la audición y de las demás modalidades, que combinadas conformarían el campo consciente entero. Partiendo de esta idea son muchos los neurocientíficos (Logothetis, 1998; Kanwisher, 2001; Navajas, Rey y Quian Quiroga, 2014; Quian Quiroga, Reddy, Kreiman, Koch \& Fried, 2005; Quian Quiroga, Mukamel, Isham, Malach \& Fried, 2008), que para estudiar la conciencia se han dedicado a estudiar la percepción visual consciente de objetos, pues estudios recientes en humanos implantados con electrodos intracraneales por motivos clínicos, han mostrado la presencia de neuronas que disparan exclusivamente cuando las imágenes son percibidas conscientemente (Sekar, Findley, Poeppel y Llinas, 2013).

En este sentido, se sabe que la vía dorsal, que va desde el área visual primaria hacia la corteza asociativa parietal, es capaz de localizar los objetos en el espacio y es inconsciente. Mientras que la vía ventral que se dirige a las áreas asociativas temporales es consciente. Las proyecciones de la corteza parietal a las áreas promotoras son inconscientes, mientras que las proyecciones de la corteza parietal a la corteza prefrontal están relacionadas con la conciencia. También sabemos que existen diversos niveles de conciencia y que la transición de la inconsciencia a la conciencia no es simplemente un cambio de una inactividad a una actividad neuronal, sino que supone un cambio en lo que hacen las neuronas, pero todavía desconocemos en qué consiste dicho cambio.

En el mismo contexto se ubica la propuesta de Bartels y Zeki (Bartels y Zeki, 1998; Zeki, 2007a, 2007b; Zeki y Bartels, 1998) denominada teoría de etapas múltiples, que considera que la actividad vinculante del cerebro no genera una experiencia consciente unificada, sino que se trata de una actividad que reúne a un conjunto de experiencias ya conscientes. Para ellos la conciencia no es una facultad unitaria sino que está formada por muchas microconciencias, que conforman el campo consciente total. "La actividad celular vinculante en nodos diferentes no es, pues, un proceso que preceda o incluso que facilite la experiencia consciente, sino que reúne diferentes experiencias conscientes" (Bartels y Zeki, 1998, p. 2330).

Hay distintas líneas de investigación que apoyan la teoría de los bloques, como los trabajos sobre la visión ciega
(Stoerig y Cowey, 1997; Weiskrantz, 1986;), la rivalidad binocular e intercambio Gestalt (Logothetis, 1998; Logothetis y Schall, 1989; Van Boxtel y Koch, 2012) y los correlatos neurales de la visión (Capurro y Quian Quiroga, 2009; Crick y Koch, 1995, 1998; Koch, 2005).

La propuesta de Crick (1994) constituye uno de los primeros enfoques reduccionistas de los procesos mentales a fenómenos neurobiológicos. Este autor sostiene que las alegrías, aflicciones, recuerdos, ambiciones y el sentido de identidad personal de cualquier individuo son el reflejo de la conducta de un ensamblaje de células y moléculas asociadas, así como que la conciencia requiere de la actividad de varias áreas corticales y del tálamo. Partiendo de este enfoque, en la actualidad parece haber cierto consenso en la comunidad neurocientífica respecto a que los fenómenos colectivos de sincronización neuronal podrían ser el mecanismo biológico específico que subyace a la conciencia.

En la visión hay una vía que va desde la retina a la corteza visual primaria, pasando por el núcleo geniculado lateral; esta vía no implica consciencia. Desde la corteza visual primaria, la información se dirige a las áreas corticales donde está representado el movimiento y a otras áreas en las que se representa el color. De ahí, la información pasa a células que reconocen los objetos en la corteza asociativa temporal inferior, donde la información se hace consciente.

Otra forma de abordar la cuestión se encuentra en la teoría del campo unificado de la conciencia. Desde esta perspectiva se trata de estudiar las diferencias entre el estado de inconsciencia (el sueño, por ejemplo) y el estado de vigilia que es la conciencia básica o de transfondo; estas diferencias constituyen el NCC que se pretende buscar. Así, la experiencia visual se considera como una modificación del campo consciente, es decir, como una nueva forma que el campo unificado adquiere; la conciencia es modulada más que generada por los sentidos (Llinas, 2008; Llinas, Ribary, Contreras y Pedroarena, 1998). Las experiencias conscientes se dan en campos unificados, de forma que para tener una experiencia visual, el sujeto tiene que estar ya consciente y la experiencia es una modificación del campo.

Para los partidarios de este planteamiento no existe, por ejemplo, una conciencia visual separada ya que sólo un sujeto ya consciente puede tener experiencias visuales por lo que la introducción de estas experiencia no es una introducción de conciencia sino la modificación de la conciencia ya preexistente. Por lo tanto no parece efectivo, considerar que la búsqueda del NCC de la visión nos pueda aportar la solución al problema que nos ocupa.

Si no se puede explicar el fenómeno general de la subjetividad cualitativa unificada recurriendo a NCC locales y específicos, entonces se debe buscar la forma en que el sistema visual introduce experiencias visuales en un campo consciente unificado ya existente. Para ello en primer lugar deberíamos conocer cómo el cerebro crea ese campo unificado consciente, es decir buscar cómo las características de un sistema formado por billones de elementos (neuronas) conectados por sinapsis, pueden producir un campo consciente. 
Se trata pues, de buscar una cierta actividad masiva del cerebro capaz de producir una experiencia consciente holística y unificada. Existen evidencias para creer que la actividad en el sistema tálamocortical es probablemente el lugar donde se debe buscar la conciencia de campo unificado.

Sólo existe un único campo consciente unificado que contiene aspectos visuales, auditivos, etcétera, por lo que la conciencia visual no se localiza en el sistema visual, ni la conciencia auditiva en el sistema auditivo. La producción de cualquier estado de conciencia por el cerebro, es la producción de una conciencia unificada. En el caso de que se confirmara esta hipótesis, quedaría resuelto el problema del vínculo de la conciencia y habría que buscar la conciencia como una característica del cerebro que emerge de las actividades de grandes masas de neuronas, que no puede ser explicada por las actividades de neuronas individuales.

Entre las diversas teorías elaboradas en el contexto al que nos estamos refiriendo, podemos destacar dos líneas contemporáneas de investigación, las llevadas a cabo por Llinas y sus colaboradores (Llinas, 1990, 2008; Llinas, et al., 1998) y por el grupo de Edelman y Tononi (Edelman, 2003; Tononi y Edelman, 1998; Tononi, Edelman y Sporns, 1998; Tononi, Sporns y Edelman, 1992).

Llinas y su grupo creen que el NCC es una actividad oscilatoria sincronizada en el sistema talamocortical. No se debe pensar en la conciencia como si fuera producida por datos sensoriales de entrada sino como un estado funcional de grandes porciones del cerebro. Los datos sensoriales de entrada sirven para modular una conciencia preexistente más que para crear de nuevo la conciencia; la conciencia no es una respuesta a estímulos sensoriales de entrada sino un estado intrínseco del cerebro. Prestan especial atención al sueño, en el que consideran que el cerebro está consciente pero es incapaz de percibir el mundo externo a través de datos sensoriales de entrada.

Para el equipo de Llinas, los fenómenos colectivos de actividad neuronal son fenómenos masivos que se originan por la excitación e inhibición simultánea y ordenada de numerosas neuronas ubicadas en redes. Existen muchos fenómenos de este tipo, entre ellos se destacan la resonancia (coincidencia de las frecuencias de oscilación en diferentes redes neuronales que da lugar al reconocimiento de un objeto) y la sincronización (ausencia de coincidencia en dicha frecuencia en dos o más conjuntos específicos de neuronas que reduce la estabilización de conexiones entre las correspondientes redes).

Llinas $(2001,2008)$ sugiere que la conciencia se produce, por la coincidencia temporal de gran escala en la actividad de las redes tálamocorticales. Esta actividad que está programada genéticamente, generaría los eventos internos como pensar, imaginar o recordar. Así pues, la arquitectura neuronal básica de la conciencia estaría formada por los ciclos de activación tálamocorticales bidireccionales que generan una actividad en las columnas resonantes de neuronas.

Por su parte, los neurobiólogos Edelman, premio Nobel de Fisiología y Medicina en 1972 por sus trabajos sobre el sistema inmunológico, y Tononi, proponen que la conciencia emerge cuando grandes grupos de neuronas forman un núcleo dinámico en el cerebro con conexiones que forman bucles entre la corteza y el tálamo. Edelman (2003) denomina a estas conexiones mapas de reentrada; se trata de algo parecido a lo que Humprey (2000), psicólogo británico, denomina bucles de realimentación reverberantes sensoriales. En ambos planteamientos subyace la idea de que el cerebro se refiere a sí mismo y es esto lo que desencadena la conciencia.

Tononi y Edelman (1998) han presentado un planteamiento al que denominan la hipótesis del núcleo dinámico. En ella la conciencia tiene dos propiedades que se dan dentro de cualquier campo consciente: la unidad y la diferenciación o complejidad (Izhikevich, et al., 2004; Sporns, Tononi y Edelman, 2000). Buscan el NCC de la unidad de la conciencia en la rápida integración que se consigue a través de los mecanismos de reentrada en el sistema tálamocortical. Para explicar la combinación de integración y diferenciación en cualquier campo consciente es preciso identificar grandes grupos de neuronas que funcionen conjuntamente (Tononi, 2002; Tononi y Edelman, 2000; Tononi y Koch, 2008), es decir, que disparen de forma sincronizada, ampliamente distribuidas por todo el cerebro, por lo que ningún área concreta y única del cerebro es responsable de la experiencia consciente.

Denominan grupo funcional a este conjunto de neuronas que disparan sincronizadamente; se trata de un grupo que debe mostrar una gran diferenciación en los elementos que lo componen para explicar los diferentes elementos de la conciencia. Los disparos sincrónicos en las regiones corticales ubicadas entre la corteza y el tálamo son indicadores directos de este grupo funcional.

Una vez identificado el grupo funcional, se trata de investigar si contiene o no en su interior, diferentes patrones de actividad de estados neuronales. La combinación de grupos funcionales junto con la diferenciación conforma la hipótesis del núcleo dinámico de la conciencia. Un núcleo dinámico está constituido por un proceso neuronal unificado de gran complejidad, que se encuentra extendido fundamentalmente en las regiones tálamocorticales, especialmente en las involucradas en la categorización perceptiva y en las que contienen los mecanismos de reentrada que ya había estudiado Edelman (1989, 1992).

Para explicar el hecho de que unas descargas neuronales de una región asociativa de la corteza puedan ir acompañadas de consciencia y otras no, Edelman y Tononi (2002) recurren a lo que denominan darwinismo neural. Ambos autores consideran que los grandes grupos de neuronas se encuentran en una situación de cooperación y competición, las que salen triunfantes de esta competición son las que van acompañadas de consciencia.

El darwinismo neural que sirve de base para la propuesta de Edelman $(1987,1989,1992)$ consiste en seleccionar dentro del sistema nervioso del grupo de neuronas el más viable mediante tres mecanismos básicos denominados: selección de desarrollo, selección experiencial y el reingreso o mapas 
reentrantes. A través de estos mecanismos se efectúa una selección de grupos neuronales específicos: por medio de la selección de desarrollo se individualiza una distribución específica de grupos de neuronas en un sistema nervioso igualmente específico; luego, a través de de la selección experiencial se favorecen relaciones específicas de grupos neuronales por medio del fortalecimiento diferencial; y, finalmente por medio de bucles reentrantes o de reingreso se actualiza efectivamente una red de relaciones de coactivación de mapas neuronales o grupo de grupos neuronales de niveles de complejidad creciente (mapas de mapas) (Izhikevich, et al., 2004; Tononi, 2002; Tononi y Edelman,1998; Tononi et al., 1992).

Estos mecanismos actúan como principios generales del funcionamiento cerebral y sirven a Edelman como base para conceptualizar la conciencia, que consiste en la integración de la conciencia primaria y la conciencia de orden superior.

Desde esta teoría se buscan los NCC de la conciencia de una manera similar a como se hace en los estudios sobre rivalidad binocular, por lo que se puede considerar que este enfoque combina características tanto de la teoría de los bloques como de la aproximación del campo unificado. Algo similar ocurre con la mayor parte de los planteamientos de los científicos que han abordado la prometedora tarea de buscar los correlatos neuronales específicos de la conciencia, como veremos a continuación.

Penrose (1989) niega que la conciencia sea un proceso computacional de las neuronas y afirma que la conciencia es reductible a fenómenos cuánticos, por lo que se trata de un reduccionismo más bien físico que neurológico. Se explica la conciencia como un producto de computaciones cuánticas en los microtúbulos del citoesqueleto neuronal. Hameroff y Penrose $(1996,2014)$ sostienen que los microtúbulos, que se encuentran en toda célula nerviosa, están diseñados para permitir la coherencia cuántica y las conexiones cuánticas en todo el cerebro. Un punto débil de esta teoría es que no explica cómo surge la experiencia subjetiva, por lo que muchos autores consideran que la teoría cuántica de la conciencia sustituye un misterio por otro. El propio Penrose (1994) también opina que la explicación del fenómeno de la vida mental requiere un conocimiento de la física que aún no tenemos.

Los neurocientíficos Friston y Frackowiak (2004) mostraron que la corteza prefrontal y el área motriz suplementaria, disminuyen su actividad cuando una acción motriz es aprendida, lo que puede indicar que estas regiones cerebrales están implicadas en la producción de conciencia. Sabemos que la corteza prefrontal está implicada en la toma de decisiones y el área motriz suplementaria en la iniciación de la acción; probablemente la corteza parietal posterior y el cerebelo sean las regiones que participan en el control inconsciente de la actividad motriz.

Bogen (1995), neurocirujano, expuso que dos de sus pacientes que tras una operación sólo habían conservado el hemisferio derecho, perdieron las funciones sensoriales y motoras de la parte derecha del cuerpo y casi toda la capacidad de hablar, pero los sujetos estaban conscientes y res- pondían adecuadamente a los estímulos. Este autor localiza el correlato neuronal de la conciencia en los núcleos intralaminares de tálamo.

Las investigaciones llevadas a cabo con pacientes aquejados de diferentes lesiones cerebrales no aportan mucha luz sobre el asunto. Las únicas lesiones cerebrales localizadas que tienen como resultado la pérdida de consciencia son las que afectan al sistema reticular de activación, situado en las porciones superiores del tronco cerebral (protuberancia y mesencéfalo) hasta el hipotálamo posterior, los núcleos talámicos intralaminares y reticulares y el cerebro basal anterior. Aunque su actividad sea esencial para el mantenimiento del estado de consciencia, se supone que por sí mismos no generan conciencia.

Se han identificado, en humanos varios correlatos de la conciencia: el bucle tálamo-cortical, un EEG característico de ondas frecuentes y de baja amplitud que va de $12-70 \mathrm{~Hz}$ y la formación reticular mesencefálica. También se ha propuesto que la descarga sincrónica de neuronas corticales, con una frecuencia de $40 \mathrm{~Hz}$, también conocida como oscilación gamma, es el correlato neural de la conciencia y sirve para unir la actividad de diversas áreas corticales en relación con un mismo objeto, aunque estudios recientes en sujetos anestesiados han mostrado que la frecuencia de $40 \mathrm{~Hz}$ puede existir sin consciencia. Además se ha postulado que las células piramidales de las capas V y VI de la corteza, cuyos axones proyectan fuera de la corteza, son responsables de la conciencia visual (Rubia, 2010).

Sirvan los diferentes planteamientos a los que hemos hecho referencia como la expresión del gran número y de la heterogeneidad de las múltiples teorías que han sido propuestas para la localización cerebral de la conciencia.

\section{Una valoración crítica a modo de conclusión.}

Los resultados de los trabajos sobre los correlatos neuronales, indican claramente que la conciencia es un producto de la actividad cerebral, pero también ponen de manifiesto que muchas de las actividades de las neuronas cerebrales no van acompañadas de consciencia. En cierta medida desde la neurociencia se ha logrado identificar los componentes bioquímicos elementales del cerebro pero también se ha puesto de manifiesto la incapacidad, por ahora, para explicar el modo en que se engendra la experiencia subjetiva. Como afirma Koch, "No se ha alcanzado todavía tanta comprensión de los procesos internos del cerebro como sería necesaria para deletrear el modo en que se engendra la conciencia a partir de la actividad química y eléctrica de las neuronas" (Koch y Greenfield, 2007, p. 50).

Además, los intentos de los neurocientíficos de dar cuenta de los fenómenos mentales y de la conducta humana apelando al cerebro, a sus estructuras y funcionamiento como el fundamento de todo el comportamiento humano no están exentos de limitaciones y pueden ser objeto de bastantes objeciones críticas. 
La cuestión relativa a si la mente puede funcionar en paralelo (de forma distribuida) u opera secuencialmente a nivel de conciencia, sigue siendo un tema vigente, independientemente de que seamos capaces de codificar perceptualmente más de un mensaje a la vez o podamos retener en la memoria a corto plazo siete elementos más o menos.

Algunos planteamientos sostienen que en cada momento sólo generamos una construcción consciente de un evento concreto que responde al propósito o tarea del momento; desde esta perspectiva se entiende que la forma de operar se podría considerar más bien serial que paralela, aunque ambos conceptos no se tomen en sentido estricto.

Si se entiende la conciencia como una construcción integrada de la evidencia disponible en cada momento que fenomenológicamente parece una totalidad, probablemente la limitación a un número de elementos se dé en las estructuras que determinan la experiencia consciente holística (Mandler, 1992). Este esquema representado en la construcción consciente debe estar restringido a un número limitado de rasgos o relaciones y estas agrupaciones cognitivas operan como las unidades de la experiencia construida como un todo integrado, como una totalidad organizada. Por lo tanto sólo un número limitado de estas agrupaciones puede formar parte de la experiencia consciente actual. Esta característica de la conciencia hace que la mente y el sistema cognitivo humano no sean desbordados por la gran cantidad de estímulos y de información que dimanan del entorno que muchas veces es irrelevante para la ejecución eficaz de los planes del momento. Diferentes trabajos de investigación, sobre todo en el campo del lenguaje, ilustran experimentalmente este carácter limitado y secuencial de la conciencia (Baars, 1988; Crick y Koch, 1990; Koch, 2009; Marcel, 1980; McKay y Bever, 1967; Rogers y McClelland, 2014; Swinney, 1979).

Los procesos conscientes controlados se diferencian de los automáticos, es decir no mediados por la conciencia, en que los primeros funcionan en serie y los segundos pueden proceder en paralelo (Froufe, 1997). En los primeros, la ejecución de un proceso implica en sí misma la inhibición de los demás, sin embargo, en los procesos automáticos, no controlados por la conciencia, no sucede lo mismo ya que la ejecución de un proceso no supone ni la inhibición de los demás ni la interferencia entre ellos. Cuando la conciencia desarrolla una construcción determinada lo hace a costa de excluir otras, por lo menos en ese momento.

Es precisamente este funcionamiento distribuido o en paralelo que caracteriza a los actuales modelos conexionistas el que genera la dificultad para seleccionar los eventos que son atendidos y cuáles no y esto supone un obstáculo para implementar la conciencia en estos sistemas si la entendemos como experiencia fenoménica y como mecanismo de funcionamiento serial.

Otro de los problemas conceptuales que debe tratar de resolver el conexionismo se refiere a la conjunción o integración de la información (binding problem) que es procesada por grupos independientes de neuronas, es lo que muchos autores denominan el problema del vínculo de la conciencia.
Se trata de dilucidar cómo se integra la información inicialmente representada de manera distribuida por billones de neuronas, cuando las diferentes características de un estímulo son procesadas de manera completamente separada por diferentes unidades, categorías o modalidades de representación (Bayne, y Chalmers, 2003). O lo que es lo mismo, de discernir cómo la información procesada de manera completamente separada llega a ser experimentada conscientemente como una sola unidad perceptual.

En el caso de la visión, por ejemplo, cuando vemos un objeto la información que llega a la retina es procesada por grupos independientes de neuronas, localizadas en distintas áreas de la corteza visual, que se han especializado para detectar colores, orientación, movimiento, etcétera. El desafío consiste en comprender cómo los disparos individuales de las neuronas en cuestión pueden dar lugar a la sensación unificada de ver el objeto estimular, es decir a la "unidad de la apercepción” (Churchland, 1994; McFadden, 2002). El cerebro procesa diferentes aspectos de la información visual (bordes, color, movimiento, ubicación relativa, etcétera) en áreas corticales separadas y cada una de ellas con distintas rutas neuronales y a pesar de que no hay un área en la que toda esta información converja y donde ocurra el intercambio de la información requerida, percibimos que nuestra experiencia se produce en torno a objetos coherentes y unitarios. Consecuentemente se supone que debe haber algún mecanismo mediante el cual todas estas informaciones se vinculan.

Todas las experiencias conscientes forman parte de un campo consciente unificado (Searle, 2002), y la importancia de la unidad se pone especialmente de manifiesto en dos áreas de investigación: los estudios de pacientes con cerebros divididos (Gazzaniga, 1988, 1998; Gazzaniga y Heatherton, 2008; Gazzaniga y Mangun, 2014) y el estudio del problema del vínculo.

La evidencia tanto anatómica como conductual de los trabajos de investigación en pacientes con cerebros divididos, sugiere que en estos sujetos hay dos centros de conciencia que después de la comisurotomía se comunican entre sí sólo imperfectamente; se trata de pacientes que parecen tener dos mentes conscientes dentro de un solo cerebro.

Las investigaciones sobre el problema del vínculo sugieren que el cerebro entero podría unificar toda la variedad de los diferentes estímulos de entrada en una sola experiencia consciente unificada. Son varios los investigadores que han explorado el papel de los disparos neuronales sincronizados en un rango de 40 Hertz para explicar la capacidad de diferentes sistemas perceptivos para vincular los diversos estímulos de neuronas anatómicamente distintas en una sola experiencia perceptiva (Llinas, 1990, 2008; Llinas y Pare, 1991; Llinas y Ribary, 1993; Singer, 1993; Singer y Gray, 1995). Otros sostienen que la unidad perceptual de la actividad consciente es una manifestación de un campo de fuerza, que podría ser electromagnético por ejemplo, capaz de enlazar e integrar la información que está distribuida en una po- 
blación de neuronas (Hardcastle, 1994; Libet, 1994, 1996; McFadden, 2002; Popper, Lindahl y Arhem, 1993).

Crick (1994) extendió la propuesta del problema del vínculo a una hipótesis general sobre los correlatos neuronales de la conciencia, la teoría de la sincronía temporal de la onda de 40 Hertz (Crick y Koch, 1990, 2007; Churchland, 1996; Koch, 1996, 2009; Koch y Segev, 1998). Un factor importante es la función integradora de las oscilaciones de 40 Hertz que se propagan a lo largo del sistema de proyecciones que se conectan bidireccionalmente desde el tálamo hasta la corteza (Crick, 1994; Crick y Koch, 1990; Edelman, 1989; Koch y Crick, 2001; Llinas, 2008; Llinas y Ribary, 1993; Llinás, Ribary, Joliot y Wang, 1994). Esta propuesta ofrece una base conceptual para poder entender de manera empírica y formal la naturaleza de la visión unificada del mundo que parece caracterizar a los fenómenos conscientes (Churchland, 1994; Chalmers, 2000; 2004, 2010).

La teoría de Llinas (2001, 2008), en primera instancia se dirige a solucionar el binding problem. En esta propuesta se considera que la unión no es espacial sino temporal y se explica sobre la base de la sincronización temporal de las descargas neuronales, no mediante la interconexión de estructuras. Así, la sincronización de otros grupos de neuronas permite que el objeto que emergió por la actividad tálamocortical específica se integre con otros objetos dentro de una experiencia fenoménica globalmente coherente. Esta definición del problema del vínculo en términos puramente temporal-funcionales de naturaleza computacional o neuronal, proporciona ideas interesantes acerca de la unión de la información y podría pasar a formar parte de una interesante teoría global de la percepción y la atención, aunque tampoco nos explica las razones por las que la sincronía neuronal va acompañada de experiencia consciente.

Por otra parte, se ha cuestionado la postura localizacionista que se deriva del uso de las modernas técnicas de imagen cerebral (Uttal, 2004) por diversas razones entre las que se pueden destacar la falta de consenso en la utilización de los constructos hipotéticos en las neurociencias; la no linealidad del sistema cerebral debida a su complejidad que desemboca en la imposibilidad de realización de análisis en unidades funcionales independientes; y el dudoso criterio estadístico a partir del que se determinan ciertos umbrales de actuación que luego son promediados por lo que se deja de lado información de ciertas áreas cerebrales correlacionadas.

En la misma línea, conviene recordar que las técnicas desarrolladas por la neurociencia sólo nos proporcionan una información parcial acerca de los fenómenos estudiados.

Así, las técnicas de neuroimagen nos aportan información de gran resolución espacial sobre la activación cerebral, pero carecen de suficiente resolución temporal, pues se requieren más de 15 segundos para obtener una imagen funcional. Es evidente que 15 segundos es un grano temporal muy grueso ya que el flujo de información cerebral (y consecuentemente de los procesos cognitivos) es muy rápido. De hecho los estudios cronométricos convencionales de la psicología nos muestran que muchos procesos significativos ocurren en tiempos muy inferiores a un segundo. Por su parte el registro electrofisiológico de ERP tiene una magnífica resolución temporal del orden de milisegundos, pero en cambio no ofrece suficiente resolución espacial (De Vega, 1998, p. 39).

Otros autores (Vul, Harris, Winkielman y Pashler, 2009) tras analizar numerosos artículos basados sobre todo en técnicas de resonancia magnética cerebral constatan que una parte importante de la investigación emplea métodos seriamente engañosos y sesgados. Además debemos tener en cuenta que las pruebas de imágenes cerebrales sólo pueden realizar mediciones indirectas del cerebro, que el empleo de colores exagera los efectos reales en el cerebro, que las imágenes cerebrales son recopilaciones estadísticas y no fotografías en tiempo real y que las áreas cerebrales se activan por varias razones. En este sentido se puede considerar que las neuroimágenes de la activación del cerebro pueden ser ambiguas y susceptibles de muchas posibles interpretaciones, por lo tanto las imágenes obtenidas con estas técnicas tienen un valor únicamente de correlación entre el comportamiento y la función cerebral (Fletcher, 2004). "A pesar de toda la publicidad en torno al desarrollo de técnicas de creación de imágenes, aún no hemos encontrado, hasta donde sé, una manera de obtener imágenes del NCC" (Searle, 2007, p. 20).

Que diferentes actividades cognitivas y comportamientos provoquen que, con las técnicas de neuroimagen, ciertas zonas cerebrales cambien de color en la pantalla digital no implica que estas áreas sean las causantes de tales comportamientos, sino simplemente que son su correlato. No se trata de un problema de las técnicas utilizadas sino del uso que hacemos de ellas y de las conclusiones que extraemos del mismo.

La pretensión de reducir el comportamiento humano a sus correlatos biológicos en el cerebro, medidos con tomografías axiales computarizadas (TAC) o con tomografías por emisión de positrones (PET) o imágenes por resonancia magnética (IRM) o cerebrografías de flujo sanguíneo cerebral regional (RCBT) o tomografías simples por emisión de fotones (SPECT), o hipotetizados como mezclas, excesos o déficit de serotonina, dopamina, noradrenalina...olvidando los objetivos de los sujetos, sus circunstancias biográficas y contextuales o su propia historia de aprendizaje, es un error tan grande como lo sería explicar la guillotina citando las leyes de la gravitación universal de Newton, sin prejuicio de que las leyes de Newton se prueben con una guillotina en funcionamiento; sin embargo, su función no es demostrar esas leyes (Castañón y Láez, 2009, p. 62).

Además existe un acuerdo bastante generalizado en considerar a la conciencia como un fenómeno psicológico que emerge del funcionamiento integrado del cerebro en el que está involucrada la intervención interactiva de múltiples estructuras nerviosas y la actividad de billones de neuronas que constantemente modifican su nivel de activación (Baars, 1988; Crick y Koch, 1990; Koch, 2009). Cada vez hay más evidencia neurofisiológica que indica que el desarrollo men- 
tal de contenidos conscientes coherentes implica la cooperación de grandes poblaciones de neuronas ampliamente distribuidas por todo el cerebro (Bogen, 1995; Crick y Koch, 1995; John, Easton y Isenhart, 1997). Si la conciencia es un fenómeno emergente de la actividad sistémica del cerebro (Froufe, 1984), como todo fenómeno emergente, tiene la capacidad de influir retroactivamente en el sistema del que emerge (Sperry, 1976), aunque cabe la posibilidad de que esta influencia se lleve a cabo a través de otros elementos o partes del sistema (Bunge, 1985).

Parece claro que cada modificación de una función mental está precedida o va acompañada de una modificación material del organismo (cerebro), pero esto sólo implica que existe una correlación entre la estructura del cerebro o la bioquímica cerebral con los fenómenos mentales o con diferentes comportamientos, pero no se puede deducir que las áreas cerebrales en cuestión sean las responsables exclusivas de los citados fenómenos o comportamientos. Así, el cerebro sería un factor necesario para la realización de tales funciones, pero no existe ninguna razón para afirmar que sea su causa.

Sin lugar a dudas, desde la teoría de los bloques se han logrado avances significativos en las investigaciones realizadas para identificar los NCC de la conciencia. Pero toda esta investigación ha sido llevada a cabo con sujetos que ya estaban conscientes, independientemente del NCC que se proponga. En el caso de la visión ciega, los pacientes analizados sólo manifiestan visión ciega si ya están conscientes, por lo que supone un problema investigar la conciencia general estudiando las diferencias entre sujetos con visión ciega y sujetos con visión normal, ya que ambos tipos de pacientes ya estaban totalmente conscientes. Lo mismo ocurre con los experimentos de rivalidad binocular e intercambio Gestalt y con los estudios sobre los correlatos neurales de la visión. En consecuencia, estas investigaciones, no nos permiten comprender las diferencias entre el cerebro consciente y el cerebro inconsciente, ni cómo se produce la conciencia en el cerebro. Desde la teoría de los bloques se supone que un sujeto totalmente inconsciente cuando se pone en funcionamiento el NCC, tendría una experiencia consciente, pero es esta una cuestión que todavía carece del respaldo de la investigación científica.

Estos planteamientos, en la casi totalidad de los casos, no se limitan a describir correlaciones cerebro-conciencia, sino que pretenden explicar la producción o emergencia de la conciencia a partir de fenómenos no conscientes, como, por ejemplo las activaciones de neuronas individuales y organizadas en redes. En este sentido Chalmers (2000) considera que una ciencia de la conciencia como máximo puede aspirar a encontrar un paralelismo entre los fenómenos observados y descritos por los neurólogos (tercera persona) y nuestras experiencias (primera persona), por lo que no considera los procesos de activación neuronal como el sustrato que produce la conciencia, sino como su correlato únicamente.

Posiblemente, como señala Searle (2007), sería más adecuado que tras encontrar los NCC de la conciencia se pruebe si la correlación es una relación causal genuina, es decir si este NCC es necesario y suficiente para la existencia de la conciencia investigando si la manipulación del NCC provoca modificaciones o pérdida de la conciencia y si un sujeto inconsciente se puede tornar consciente al inducir el supuesto NCC, para desarrollar un conjunto de leyes que formalicen una teoría de las relaciones causales. Se trataría pues de probar que el NCC no solo está correlacionado con la conciencia, sino que es causalmente necesario y suficiente para la presencia de la misma.

En los últimos años, se han intentado explicar múltiples comportamientos recurriendo, de forma excesiva desde mi punto de vista, a las estructuras cerebrales, a la bioquímica cerebral o a la genética. Así, por ejemplo, para explicar los afectos positivos ante un acontecimiento regocijante o las emociones negativas ante un suceso decepcionante se ha apelado exclusivamente a los niveles de serotonina en el sistema nervioso central, afirmando que las emociones negativas son debidas a los bajos niveles de este neurotransmisor y las emociones positivas son consecuencia de los altos niveles de serotonina en el sistema nervioso. Posiblemente no sea el sistema nervioso con sus neurotransmisores el que causa las emociones positivas ante un acontecimiento regocijante o negativas ante uno decepcionante, aunque el organismo se sirva de los neurotransmisores para experimentar estas emociones ante acontecimientos de diferente índole. ¿La tristeza está causada por la escasez de serotonina o los niveles de serotonina son bajos porque estamos tristes a consecuencia de un suceso decepcionante? En la misma línea de excesos se ha pretendido explicar desde esta perspectiva el comportamiento amoroso, el misticismo, las experiencias religiosas, los comportamientos políticos, o la neurología de la intencionalidad.

No se pueden explicar los fenómenos mentales apelando exclusivamente a las estructuras cerebrales. El análisis psicológico del comportamiento humano debe tener en cuenta los factores biológicos pero no considerándolos como los únicos elementos causales del mismo sino como aspectos facilitadotes o inhibidores. Thompson (2007) considera que la actividad neuronal no explica el complejo comportamiento de los organismos, pero puede contribuir dando cuenta de la variabilidad conductual relacionada con los casos en los que se producen desórdenes comportamentales relacionados con alteraciones biológicas, cerebrales o genéticas y con el déficit en la producción de neurotransmisores. Estos desórdenes que implican pobres conexiones neuronales están en la base del desarrollo de comportamientos desadaptativos. En este sentido se establecen relaciones entre genes, funciones mentales, estructura cerebral y comportamiento, ahora bien, se trata de una relación que toma los elementos neurales como factores disposicionales, es decir, como elementos que pueden facilitar o dificultar ciertas interacciones (Ryle, 2005).

No cabe duda de que la genética, la química cerebral, la fisiología del cerebro y otros factores relacionados desempeñan un importante papel en la comprensión de la conducta ya que la relación entre el organismo, el comportamiento y 
los aspectos del mundo con los que el individuo interacciona tienen como base la dotación genética y el funcionamiento cerebral, pero explicar todos los comportamientos y fenómenos mentales exclusivamente en base a mecanismo biológicos, reacciones químicas, etcétera, es conceder excesiva importancia al determinismo biológico, lo que supone un reduccionismo radical. El comportamiento humano tiene correlatos cerebrales que en ningún caso lo explican; el funcionamiento cerebral es necesario pero insuficiente para dar cuenta del comportamiento humano.

En cualquier caso, en el seno de las ciencias cognitivas las aportaciones de las neurociencias constituyen formulaciones de gran utilidad para la investigación psicológica. Los datos ofrecidos por las neurociencias requieren explicaciones algorítmicas, funcionales o de otro tipo, para lograr una plena comprensión de los fenómenos mentales por lo que son datos que estimulan el análisis funcional de la mente. Aunque sabemos que las técnicas desarrolladas por la neurociencia nos proporcionan una información parcial sobre los fe-

\section{Referencias}

Abbott, L. y Sejnowski, T.J. (Eds.) (1999). Neural codes and distributed representations: foundations of neural computation. Cambridge, MA: MIT Press.

Baars, B. (1988). A cognitive theory of consciousness. New York, NY: Cambridge University Press.

Bartels, A. y Zeki, S. (1998). The theory of multistage integration in the visual brain. Proceedings of the Royal Society B, 265, 2327-2332.

Bayne, T. y Chalmers, D. J. (2003). What is the Unity of consciousness? En A.Cleeremans (ed.), The Unity of Consciousness: Binding, Integration, Dissociation (pp.23-58). New York, NY: Oxford University Press.

Bogen, J. E. (1995). On the neurophysiology of consciousness: I. An overview. Consciousness and Cognition, 4, 52-62.

Bunge, M (1985). El problema mente-cerebro: un enfoque psicobiológico. The problema mind-brain: a psychobiological approach7. Madrid: Tecnos.

Capurro, A. y Quian Quiroga, R. (2009). The neural correlates of perceptual awareness. Psyche, 15, 29-38.

Castañón, M. y Láez, M. C. (2009). Psicología y Neurociencias. Buscar la llave donde hay luz y no donde se perdió. [Psychology and Neurosciences. To find the key where is ligth and not where it was lost]. Prolepsis, 3, 6070.

Chalmers, D. J. (1997). The Concious Mind: In Search a Foundamental Theory. New York, NY: Oxford University Press.

Chalmers, D. J. (1998). On the Search for the Neural Correlate of Consciousness. En S.Hameroff. A.Kaszniak, y A.Scott, (eds.), Toward a Science of Consciousness II (pp. 219-230). Cambridge, MA: MIT Press.

Chalmers, D. J. (2000). What is a neural correlate of consciousness? En E.T.Metzinger (ed.), Neural correlates of consciousness. Conceptual and empirical question (pp.17-39). Cambridge, MA: MIT Press.

Chalmers, D. J. (2004). How can we construct a science of consciousness? En M. Gazzaniga (ed.), The cognitive Neurosciences III (pp.1111-1119). Cambridge, MA: MIT Press.

Chalmers, D. J. (2010). The character of consciousness. New York, NY: Oxford University Press.

Chalmers, D. J. (2012). The Varieties of Computation: A Reply. Journal of Cognitive Science, 13, 211-248.

Churchland, P. M. (1994). Can neurobiology teach us anything about consciousness? Proceeding and Addresses of the American Philosophical Association, $67,23-40$.

Churchland, P. M. (1996). Learning and conceptual change: The view from the neurons. En A. Clark y P .J. R. Millican (eds.), Connectionism, concepts and folk psychology: The legacy of Alan Turing (vol. 2) (pp. 7-44). Oxford, England: Clarendon Press. nómenos investigados, desde la psicología cognitiva se debe mantener una buena comunicación con esta disciplina y conocer los avances en sus técnicas de investigación. Esto no implica, en ningún caso, que los datos neurofisiológicos sustituyan a la psicología cognitiva, ya que siempre será necesario disponer de modelos interpretativos de los datos; en la medida que los datos de la neurociencia sean más minuciosos, más estructurados y precisos deberán ser los modelos psicológicos interpretativos.

Esto, no debe hacernos olvidar que la psicología todavía tiene mucho trabajo que llevar a cabo antes de que su relación con las neurociencias nos lleve al conocimiento adecuado y riguroso de la mente. Ahora bien, hoy parece muy claro que es totalmente necesario incorporar el nivel biológico para poder explicar los fenómenos psicológicos, aunque conviene recordar que la psicología cognitiva todavía no ha generado un modelo general de la cognición que sea objeto de un amplio consenso, sino varios micromodelos con un limitado poder explicativo.

Cosmides, L. y Tooby, J. (1995). From function to structure: The role of evolutionary biology and computational theories in cognitive neuroscience. En M. S. Gazzaniga (ed.), The cognitive neuroscience (pp. 1199-1210). Cambridge, MA: MIT Press.

Cosmides, L. y Tooby, J. (2013). Evolutionary psychology: New perspectives on cognition and motivation. Annual Review of Psychology, 64, 201-229.

Cotterill, R. (1998). Enchanted Looms: Consciousness Networks in Brain and Computers. Cambridge, England: Cambridge University Press.

Crick, F. (1994). The Astonishing Hipotesis: The Scientific Search for the Soul. New York, NY: Scribner.

Crick, F. y Koch, C. (1990). Towards a neurobiological theory of consciousness. Seminars in the Neurosciences, 2, 263-275.

Crick, F. y Koch, C. (1995). Are we aware of neural activity in primary visual cortex? Nature, 375, 121-123.

Crick, F. y Koch, C. (1998). Consciousness and neuroscience. Cerebral Cortex, 8(2), 97-107.

Crick, F. y Koch, C. (2003). A Framework for Consciousness. Nature Neuroscience, 6, 119-126.

Crick, F. y Koch, C. (2007). A neurobiological for Consciousness. The Blackwell Companion to Consciousness, 567-579.

Damasio, A. R. (1996). El error de Descartes [Descartes' Error]. Barcelona: Crítica.

Damasio, A. R. (1999). The Feeling of What Happens, Body and Emotion in the Making of Consciousness. New York, NY: Harcourt Brace Jovanovich.

Damasio, A. R. (2000). Creación cerebral de la mente. [Cerebral creation of the mind]. Investigación y Ciencia, 1, 66-71.

Damasio, A. R. (2001). La sensación de lo que ocurre. Cuerpo y emoción en la construcción de la conciencia. [The sensation of what happens. Body and emotion on the consciousness construction]. Madrid: Debate.

De Vega, M. (1998). La psicología cognitiva: ensayo sobre un paradigma en transformación. [The cognitive psychology: essay about a paradigm in transition]. Anuario de Psicología, 29 (2), 21-44.

Eccles, J. C. (1980). The Human Psyche. New York, NY: Springer-Verlag.

Eccles, J. C. (1989). Evolution of the Brain: Creation on the Self. New York, NY: Routledge, Chapman y Hall.

Edelman, G. M. (1987). Neural Darwinism: The Theory of Neural Group Selection. New York, NY: Basic Books.

Edelman, G. M. (1989). The Remembered Present: A Biological Theory of Consciousness. New York, NY: Basic Books.

Edelman, G. M. (1992). Bright Air; Brilliant Fire: On the Matter of the Mind. New York, NY: Basic Books. 
Edelman, G. M. (2002). Displacing metaphysics: consciousness research and the future of neuroscience. En F. Mora (ed.), Continuum: cómo funciona el cerebro (pp. 147-166). Madrid: Alianza Ensayo.

Edelman, G. M. (2003). Naturalizing consciousness: A teoretical framework. Proceedings of the National Academy of Sciences USA, 100, 5520-5524.

Edelman, G. M. y Tononi, G. (2002). El universo de la conciencia: Cómo la materia se convierte en imaginacion. The universe of the consciousness: How the substance turnes into imagination]. Barcelona: Crítica.

Fletcher, P. C. (2004). Functional neuroimagining of psychiatric disorders: exploring hidden behaviour. Psychological Medicine, 34, 577-581.

Freeman, W. J. (1995). Societies of Brains: A Study in the Neuroscience of Love and Hate. Hillsdale, NJ: Erlbaum.

Friston, K. y Frackowiak, R. (2004). Human Brain Function. San Diego, CA: Academic Press.

Froufe, M. (1984). Introspección e informes verbales en procesamiento humano de información. Introspection and verbal reports in human information processing] Estudios de Psicología, 19/20, 133-155. doi: 10.1080/02109395.1984.10821407

Froufe, M. (1997). El inconsciente cognitivo. La cara oculta de la mente. The cognitive unconscious. The bidden face of the mind]. Madrid: Biblioteca Nueva.

Gabbiani, F. y Koch, C. (1999). Coding of time-varying signals in spike trains of integrate-and-fire neurons with random threshold. En L. Abbott y T.J. Sejnowski (eds.), Neural codes and distributed representations: Foundations of neural computation (pp. 201-224). Cambridge, MA: MIT Press.

Gardner, H. (1987). La nueva ciencia de la mente. Historia de la revolución cognitiva. [The Minds New Science: A bistory of the cognitive revolution]. Buenos Aires, Argentina: Paidós.

Gazzaniga, M. S. (1988). How Mind and Brain Interact to Create Our Conscious Lives. Boston, MA: Houghton Mifflin y Cambridge, MA: MIT Press.

Gazzaniga, M. S. (1998). El pasado de la mente. [The Mind's Past]. Barcelona: Editorial Andrés Bello.

Gazzaniga, M. S. y Heatherton, T. (2008). Psychological Science: Mind, Brain and Behavior. New York, NY: W.W. Norton.

Gazzaniga, M. S. y Mangun, G. R. (2014) (eds.), Cognitive Neuroscience V. Cambridge, MA: MIT Press.

Greenfield, S. (2011). You and Me: The neuroscience of Identity. London, UK: Notting Hill Editions.

Greenfield, S. y Collins, T. F. T. (2005). A Neuroscientific Approach to Consciousness. Progress in Brain Research, 150, 11-23.

Hameroff, S. R. (1998a). Funda-Mentality: Is the conscious mind subtly linked to a basic level of the universe? Trends in Cognitive Sciences, 2(4), 119-127.

Hameroff, S. R. (1998b). Quantum computation in brain microtubules? The Penrose-Hameroff "Orch OR" model of consciousness. Philosophical Transactions of the Royal Society A, 356, 1869-1896.

Hameroff, S. R. y Penrose, R. (1996). Conscious events as orchestrated space-time selection. Journal of Conciousness Studies, 3, 36-53.

Hameroff, S. R. y Penrose, R. (2014). Consciousness in the universe: a review of the "Orch OR" theory. Physics of Life Reviews, 11(1), 39-78. doi: 10.1016/j.plrev.2013.08.002

Hardcastle, V. G. (1994). Psychology's binding problem and possible neurobiological solutions. Journal of Consciousness Studies, 1, 66-90.

Humprey, N. (2000). How to solve the mind-body problem. Thorverton, England: Imprinting Academia

Izhikevich, E. M., Gally, J. A. y Edelman, G. M. (2004). Spike-timing dynamics of neuronal groups. Cerebral Cortex, 14, 933-944.

John, E. R., Easton, P. e Isenhart, R. (1997). Consciousness and cognition maybe mediated by multiple independent coherentensembles. Consciousnes and Cognition, 6, 3-39.

Kanwisher, N. (2001). Neural events and perceptual awareness. Cognition, 79 , $89-113$

Koch, C. (1996). Toward the neuronal substrate of visual consciousness. En S.A. Hameroff, A. W. Kaszniak y A. C. Scott (eds.), Toward a science of consciousness (pp. 247-258). Cambridge, MA: MIT Press.

Koch, C. (2005). Vision: The inchoate science of consciousness. The Scientist, 19(17), 14-16

Koch, C. (2009). A Theory of Consciousness. Scientific American Mind, 20(4), 16-19.

Koch, C. (2013). The Concious Infant. Scientific American Mind, 24(4), 24-25.
Koch, C. y Segev, I. (1998). Neural modeling: From ions to networks. Cambridge, MA: MIT Press.

Koch, C. y Crick, F. (2001). The neurobiology of consciousness. Cambridge, MA: MIT Press.

Koch, C. y Tsuchiya, N. (2007). Attention and consciousness: distinct brain processes. Trends in Cognitive Sciences, 11(1), 16-22.

Koch, C. y Greenfield, S. (2007). ¿Cómo surge la conciencia? [How does consciousness happen?]. Investigación y Ciencia, 375, 50-57.

Kosslyn, S. M. y Koenig, O (1992). Wet mind: The new cognitive neuroscience. New York, NY: Free Press.

Libet, B. (1994). A testable field theory of mind-brain interaction. Journal of Consciousness Studies, 1, 119-126.

Libet, B. (1996). Conscious mind as a field. Journal of Theoretical Biology, 178, 223-226.

Libet, B. (2004). Mind time: the temporal factor in consciousness. Harvard University Press.

Llinas, R. R. (1990). Intrinsic electrical properties of mammalian neurons and CNS function. Fidea Research Foundation Neuroscience Award Lectures, 4, 1-10.

Llinas, R. R. (2001). I of the Vortex. From Neurons to Self. Cambridge, MA: MIT Press.

Llinas, R. R. (2008). Of self-awareness: The basic neuronal circuit in human consciousness and the generation of self. Journal of Consciousness Studies: Controversies in Science \&o the Humanities, 15(9), 64-74.

Llinas, R. R. y Pare, D. (1991). Of dreaming and wakefulness. Neuroscience, 44, 521-535.

Llinas, R. R. y Ribary, U. (1993). Coherent 40-Hz oscillation characterizes dream state in humans. Proceedings of the National Academy of Sciences, 90, 2078-2081.

Llinas, R. R.; Ribary, U.; Joliot, M. y Wang, X. J. (1994). Content and context in temporal thalamocortical binding. En G. Buzsaki, R.R. Llinás y W. Singer (eds.), Temporal Coding in the Brain (pp. 251-272). Berlin, Germany: Springer Verlag.

Llinas, R. R, Ribary, U., Contreras, D. y Pedroarena, C. (1998). The neuronal basis for consciousness. Philosophical Transactions of the Royal Society B, 353 , 1841-1849.

Logothetis, N. (1998). Single units and conscious vision. Philosophical Transactions of the Royal Society B, 353, 1801-1818.

Logothetis, N. y Schall, J. (1989). Neuronal correlates of subjective visual perception. Science, 245, 761-763.

Mandler, G. (1992). Toward a theory of consciousness. En H.Geissler; S. W. Link y J. T. Townsend (eds.), Cognition, information processing and psychophysics (pp. 43-65). Hillsdale, NJ: LEA.

Marcel, A. J. (1980). Conscious and preconscius recognition of polysemous words: Locating the effects of prior verbal contex. En R. S. Nickerson (ed.), Attention and perfomance, VIII (pp. 435-457). Hillsdale, NJ: Erlbaum.

Martinez, F. E. (2006). El proyecto computacional conexionista en el análisis teórico de la actividad conciente. [The conexionist computational project on the theoretical analysis of the conscious activity]. Revista Latinoamericana de Psicologia, 38(1), 137-148.

McClelland, J. L. (2010). Emergence in Cognitive Science. Topics in Cognitive Science, 2, 751-770. doi: 10.1111/j.1756.2010.01116.x

McClelland, J. L. (2013). Integrating probabilistic models of perception and interactive neural networks: a historical and tutorial review. Frontiers in Psychology, 4, 503. doi: 10.3389/fpsyg. 2013.00503

McCulloch, W. y Pitts, W. (1943). A logical calculus of the ideas inmanent in nervous activity. Bulletin for Mathematical Biophysics, 5, 115-155.

McFadden, J. (2002). Evidence for an electromagnetic field theory of consciousness. Journal of Consciousness Studies, 9, 23-50.

McKay, D. G. y Bever, T. G. (1967). In search of ambiguity. Perceptions and Psychophysics, 2, 193-200.

Navajas, J., Rey, H. J. y Quian Quiroga, R. (2014). Perceptual and contextual awareness: Methodological considerations in the search for the neural correlates of consciousness. Frontiers in Psychology, 5 ,959. doi: 10.3389/psyg.2014.00959

O`Reilly, R. C. y Munakata; Y. (2000). Computacional explorations in cognitive neuroscience: Understanding the mind by simulating the brain. Cambridge, MA: MIT Press. 
Penrose, R. (1989). The Emperor's New Mind. Oxford, England: Oxford University Press.

Penrose, R. (1994). Shadows of the mind. Oxford, England: Oxford University Press.

Popper, K. R.; Lindahl, B. I. y Arhem, P. (1993). A discussion of the mindbrain problem. Theoretical Medicine, 14, 167-180.

Pribram, K. (1976). Problems concerning the structure of consciousness. En G. Globus, G. Maxwell e I. Savodnik (eds.), Consciousness and Brain: A Scientific and Philosophical Inquiry (pp. 297-313). New York, NY: Plenum.

Pribram, K. (1991). Brain and perception: Holonomy and Structure in Figural Procesing. Hillsdale, NJ: Erlbaum.

Pribram, K. (1999). Brain and the composition of conscious experience. Journal of Consciousness Studies, 6(5), 19-42.

Quian Quiroga, R. (2008). Las neuronas de la conciencia. [The neurons of the consciousness]. Ciencia Cognitiva, 2, 47-49.

Quian Quiroga, R., Reddy, L., Kreiman, G., Koch, C. y Fried, I. (2005). Invariant visual representation by single neurons in the human brain. $\mathrm{Na}$ ture, 435, 1102-1107. doi: 10.1038/nature03687

Quian Quiroga, R., Mukamel, R., Isham, E., Malach, R. y Fried, I. (2008). Human single-neuron responses at the threshold of conscious recognition. Proceedings of the National Academy of Sciences, 105, 3599-3604.

Rogers, T. T. y McClelland, J. L. (2014). Parallel Distributed Processing at 25: Further Explorations in the Microstructure of Cognition. Cognitive Science, 38, 1024-1077. doi: 10.1111/cogs.12148

Rubia, F. J. (2010). La consciencia es el mayor enigma de la ciencia y la filosofía. [The consciousness is the biggest enigma os the science and philosophy].Pandaemonaeon, julio, 1-16.

Ruiz-Vargas, J. M. (1999). Psicología experimental versus neurociencia cognitiva: hacia una relación convergente. [Experimental psychology versus cognitive neuroscience: towards a convergence]. Escritos de Psicología, 3 , 13-19.

Rumelhart, D. McClelland, J y el PDP Research Group (1986). Parallel distributed processing: Explorations in the microstructure of cognition, Vol.1: Foundation. Cambridge, MA:MIT Press.

Ryle, G. (2005). El concepto de lo mental. [Mental concept]. Barcelona: Trillas.

Searle, J. R. (2002). Consciousness and Language. New York, NY: Cambridge University Press.

Searle, J. R. (2007). La conciencia. [The consciousness]. Diálogos de Bioética, junio, 1-34.

Sekar, K., Findley, V. M., Poeppel, D. y Llinas, R. R. (2013). Cortical response tracking the conscious experience of threshold duration visual stimuli indicates visual perception is all or none. Proceedings of the National Academy of Sciences of the United States of America, 110, 5642-5647.

Singer, W. (1993). Synchronization of cortical activity and its putative role in information processing and learning. Annual Review of Physiology, 55, 349375.

Singer, W. y Gray, C. (1995). Visual feature integration and the temporal correlation hypothesis. Annual Review of Neuroscience, 18, 555-586.

Smolensky, P. (1988). On the proper treatment of connectionism. Behavioral and Brain Sciences, 11, 1-74.

Smolensky, P. (2012). Subsimbolic computation theory for the human intuitive processor. Lecture Notes in Computer Science, 7318, 676-686.

Sperry, R. W. (1976). Mental phenomena as causal determinants in brain function. En G. Globus, G. Maxwell e I. Svodnik (eds.), Conciousness and the brain (pp. 163-177). New York, NY: Plenum Press.
Sperry, R. W. (1993). The impact and promise of the cognitive revolution. American Psychologist, 48(8), 878-885.

Sperry, R. W. (2000). Mente, cerebro y valores humanistas. [Mind, Brain, and humanist values]. Escritos de Psicología, 4, 1-7.

Sporns, O. (2013). Structure and function of complex brain networks. Dialogues in Clinical Neuroscience, 15(3), 247.

Sporns, O, Tononi, G. y Edelman, G. M. (2000). Connectivity and complexity; the relationship between neuroanatomy and brain dynamics. Neural Networks, 13, 909-922.

Stoerig, P. y Cowey, A. (1997). Blindsight in man and monkey. Brain, 12, 535-551.

Swinney, D. A. (1979). Lexical acessduring sentence comprehension: (Re)consideration of context effects. Journal of Verbal Learning and Verbal Behavior, 18, 645-659.

Thompson, T. (2007). Relations among functional systems in behavior analysis. Journal of the Experimental Analysis of Behavior, 83, 423-440.

Tononi, G. (2002). Conciencia y complejidad. [Consciousness and complexity]. En R. Adolphs, H. Eichenbaum, J. D. Delius., J. LeDoux., R. Picard, G. Tononi,. et al. (eds.), Emoción y conocimiento. La evolución del cerebro y la inteligencia (pp. 195-217). Barcelona: Tusquets Editores.

Tononi, G., Sporns, O. y Edelman, G. (1992). Reentry and the problem of integrating multiple cortical areas: simulation of dynamic integration in the visual system. Cerebral Cortex, 2, 310-335.

Tononi, G., Edelman, G. y Sporns, O. (1998). Complexity and coherency: integrating information in the brain. Trends in Cognitive Sciences, 2(12), 474-484.

Tononi, G. y Edelman, G. (1998) Consciousness and complexity. Science, 282, 1846-1851.

Tononi, G. \& Edelman, G. M. (2000). Schizophrenia and the mechanism of conscious integration. Brain Research Reviews, 31, 391-400.

Tononi, G. y Koch, C. (2008). The Neural Correlates of Consciousness. Annals of the New York Academy of Sciences, 1124(1), 239-261. doi: 10.1196/annals.144.004

Uttal, W. (2004). Hypothetical high-level cognitive functions cannot be localized in the brain: another argument for a revitalized behaviorism. The Behavior Analyst, 27, 1-6.

Van Boxtel, J. J. A. y Koch, C. (2012). Visual rivalry without spatial conflict. Psychological Science, 23(4), 410-418.

Vul, E., Harris, Ch., Winkielman, P. y Pashler, H. (2009). Puzzlingly High Correlations in fMRI Studies of Emotion, Personality, and Social Cognition. Perspectives on Psychological Science, 4, 274-290.

Weiskrantz, L. (1986). Blindsight: A case Study and Implications. New York, NY: Oxford University Press.

Zeki, S. (2007a). A theory of micro-consciousness. In M. Velmans y S. Schneider (eds.), The Blackwell companion to consciousness (pp. 580-588). Blackwell: Oxford University Press.

Zeki, S. (2007b). The disunity of consciousness. Progress in Brain Research, $168,11-18$

Zeki, S. y Bartels, A. (1998). The autonomy of the visual systems and the modularity of conscious vision. Philosophical Transactions of the Royal Society B, 353, 1911-1914.

(Articulo recibido: 17-10-2013; revisado: 25-10-2014; aceptado: 08-11-2014) 